\title{
A Framework for Adaptive Residual Streaming for Single-Player Cloud Gaming
}

\author{
DE-YU CHEN and MAGDA EL-ZARKI, University of California, Irvine
}

\begin{abstract}
Applying cloud technology to 3D interactive multimedia applications is a promising way to provide flexible and cost-efficient online high-bandwidth immersive services to a large population of end users. One main reason cloud systems are popular among users is the fact that it relaxes the hardware requirements for highend interactive visual applications. As most of the computational tasks are done on cloud servers, users no longer need to upgrade their hardware as frequently to keep up with the ever-increasing high-end computing requirements of the latest applications. Moreover, cloud systems make it easier for a user to enjoy applications on different platforms, including mobile devices that are usually not powerful enough to run high-end, memory-intensive services. In short, applying cloud technology to high-end immersive applications has advantages in cost-efficiency and flexibility both for the end users and the service providers. However, there are two main drawbacks to applying cloud technology to 3D interactive multimedia services: (1) high-bandwidth utilization and (2) latency. In this article, we propose a framework that addresses the two problems for singleplayer cloud gaming by using a combination of collaborative rendering, progressive meshes, and 3D image warping techniques. The experimental results show that the proposed system can reduce the bandwidth usage and improve the visual quality by utilizing local computing power on the client. The results also show that the interaction latency can be reduced somewhat by sacrificing some degree of visual quality in the end system.
\end{abstract}

CCS Concepts: • Information systems $\rightarrow$ Multimedia streaming; • Applied computing $\rightarrow$ Computer games; $\bullet$ Computer systems organization $\rightarrow$ Client-server architectures;

Additional Key Words and Phrases: Collaborative rendering, progressive meshes, 3D image warping

ACM Reference format:

De-Yu Chen and Magda El-Zarki. 2019. A Framework for Adaptive Residual Streaming for Single-Player Cloud Gaming. ACM Trans. Multimedia Comput. Commun. Appl. 15, 2s, Article 66 (July 2019), 23 pages.

https://doi.org/10.1145/3336498

\section{INTRODUCTION}

Cloud gaming has been considered as a solution to provide high-end interactive 3D services to a large population of gamers. As opposed to running the entire game instance locally, cloud gaming systems rely on a remote game server for doing most of the compute intensive tasks. As a result, players no longer need to upgrade their hardware as frequently to keep up with the ever-increasing high-end computing requirements of the latest games. Moreover, the resulting thin client makes it easier for players to enjoy playing games on different platforms, including mobile devices that

Authors' addresses: D.-Y. Chen and M. El-Zarki, Department of Computer Science, School of Information and Computer Science, University of California, Irvine, California 92697-3425; emails: \{teyuc, elzarki\}@uci.edu.

Permission to make digital or hard copies of all or part of this work for personal or classroom use is granted without fee provided that copies are not made or distributed for profit or commercial advantage and that copies bear this notice and the full citation on the first page. Copyrights for components of this work owned by others than ACM must be honored. Abstracting with credit is permitted. To copy otherwise, or republish, to post on servers or to redistribute to lists, requires prior specific permission and/or a fee. Request permissions from permissions@acm.org.

(C) 2019 Association for Computing Machinery.

1551-6857/2019/07-ART66 \$15.00

https://doi.org/10.1145/3336498 
are usually not powerful enough to run high-end games. In short, cloud gaming has advantages in cost-efficiency and flexibility both for the end users and the game providers.

Some recent trends in the IT industry have changed the environment that once favored cloud usage. The slowing down of Moore's law makes upgrading hardware less urgent. According to the latest news, the PC upgrade cycle has slowed down to every 5 to 6 years [1]. However, data transfer cost is still a major concern for data intensive services such as cloud gaming. Amazon EC2 now charges $\$ 0.05$ to $\$ 0.09$ for every gigabyte of data transferred over the Internet [2]. The price may not seem extremely high, but it can quickly add up with bandwidth-intensive services such as video streaming. Aside from the per unit cost for data transfer, the excessive network traffic can cause other problems such as packet loss and high latency, which impacts the users' quality of experience. Another big issue of cloud gaming is the network latency. In typical cloud gaming solutions, the client needs to wait at least a whole network round-trip time to get a response. For interactive applications like gaming, such a delay is simply too long. All of these factors combined diminish the attractiveness of the current cloud gaming paradigm for end users. On the other hand, increasingly more powerful mobile devices are becoming a viable option for gamers to enjoy highend gaming experience. The wide diversity in user end devices makes choosing the solution that best fits the needs of all end users a difficult problem for developers that want to offer a one-sizefits-all solution.

To cloud or not to cloud is a dilemma for both game service providers and gamers. However, we believe it should not be such a dichotomy. In this article, we propose a flexible way to provide cloud services to end users with different resources and quality constraints. By utilizing collaborative rendering and progressive meshes techniques, the proposed system can operate anywhere between purely local execution mode and thin-client cloud gaming mode. Choosing the appropriate mode will be a factor of the availability of resources including local/remote computing power, network bandwidth, and, of course, cost to the end user. Due to the nature of progressive meshes, the system can switch, dynamically and smoothly, between different usage cases, as the availability of resources changes over time, with the goal of reducing end-user costs, while maintaining an acceptable level of "game" experience. To address the latency issue, a 3D image warping technique is used to dynamically control interaction delay with a tradeoff in image quality, which adds another dimension of flexibility. Furthermore, our approach puts very little extra burden on the software developers and can be easily applied to existing applications, as all configuration changes are done automatically without any game content dependence.

This work is an extension of our previous work [7]. In [6], we studied the potential performance gain of buffering information in our proposed system.

The rest of the article is organized as follows: Section 2 discusses prior work related to our approach. Section 3 provides an overview of the system architecture. Section 4 outlines how collaborative rendering and progressive mesh techniques are used to achieve dynamically adjustable bandwidth reduction and presents performance measurements of our bandwidth reduction method. In Section 5, we show how 3D image warping is used to reduce interaction latency and present results of the proposed approach. We provide our conclusions highlighting our contributions and discuss future directions in Section 6.

\section{RELATED WORK}

In recent years, several cloud based interactive 3D systems have been proposed by teams in both academia and industry. Each targets a different performance goal and has different requirements on the capabilities of both the client and the server. In this section, we provide an overview of the most widely cited approaches and existing work that is closely related to our proposed system. 


\subsection{Cloud-Based Interactive 3D Systems}

Most of the existing cloud-based interactive 3D systems use gaming, due to its demanding service requirements, as their target or example application. Below we describe the three most popular approaches for cloud services: video streaming, graphics streaming, and collaborative rendering.

Video streaming is currently the most popular method used to offer online 3D immersive cloud services. In this type of system, the server is in charge of executing both the application logic and the rendering. The rendered images are encoded and streamed to the client as a video service. Most of the commercial cloud services for gaming have adopted this approach. GamingAnywhere [20] is an example of an open-source video-streaming-based cloud system. The thin-client nature of these systems makes it an ideal solution for bringing high-end immersive experiences to all kinds of devices. The main disadvantage is that video streaming consumes substantial bandwidth even with efficient encoding [9].

In graphics streaming, the server only updates the environment state. All API calls to graphics libraries such as OpenGL and DirectX are intercepted, encoded and streamed to the client. The rendering takes place on the client by executing the received graphics commands. Due to the complexity of the system, graphics streaming is not as popular as video streaming. Games@Large [32] is one of the very few systems that supports graphics streaming. Without efficient compression, the bandwidth required for streaming graphics commands can be higher than video streaming, and it fluctuates more dramatically. However, since the rendering is done locally on the client, it does not have the issue of lossy encoding and thus has less distortion in the visual quality than the video streaming method that is based on lossy compression. To address the bandwidth problem, Liao et al. proposed a few novel ideas in [24]. Surface simplification and interpolation of geometry data are used for intra-frame and inter-frame compression, respectively. In addition, a caching mechanism is proposed to minimize the bandwidth usage by frequently used commands and data.

Collaborative rendering requires even more resources on the client device. In this type of system, the server and the client both maintain a synchronized state space. The client renders a low-quality version of the application locally, and the server renders it in both high and low quality, computes the difference, and sends it as a patch to the client for generating better visual quality. Since the difference is usually small, it can be encoded efficiently. This method generally provides better visual quality with lower bandwidth costs, but at an increased cost in computation on both the server and the client. Cuervo et al. [11] proposed Kahawai, a system specifically aimed at the gaming market, that is based on collaborative rendering.

\subsection{Adaptive Cloud-Based Interactive 3D Systems}

The quality of service of all immersive and interactive cloud services depends heavily on network conditions. Various studies have addressed the issue of the dynamic and unstable nature of the Internet. Below we summarize some of the work that attempts to address the requirements of the applications, on different types of end-user platforms, at the same time aiming for best performance in the face of resource constraints that are very unpredictable and that can severely impact the quality of service.

Hong et al. [16] propose dynamic codec reconfiguration techniques to enable frame rate and bitrate adaptation in video-streaming-based systems. Shirmohammadi [37] suggests that by omitting some less important objects, the complexity of the scene can be decreased, which not only lowers the bandwidth usage but also reduces the encoding time. However, it is also shown that the importance of the objects is context dependent. Therefore, the adaptation cannot be accomplished without the additional efforts associated with object labeling, of the application developer. In [25], 
Liu et al. dynamically allocate a bit rate to macroblocks within each frame based on their importance calculated from rendering information.

Cai et al. [5] propose a more generalized adaptation framework which considers both the network and the computing resources. By decomposing the environment into a set of inter-dependent components, each component can be assigned dynamically to either the server or the client for execution, in order to meet different performance requirements. Although this framework is highly flexible, it is unlikely to be widely accepted by developers because it requires them to develop the 3D applications, such as games, in a whole new way. Besides, the computing and communication costs will most likely be dynamic and thus hard to estimate accurately.

\subsection{Other Similar and/or Hybrid Approaches}

Ahmadi et al. [3] proposed a context based visual attention model called Game Attention Model (GAM). GAM considers two different forms of human attention: (1) Bottom-up stimulus-driven attention, which is related to intensity, color, texture, etc. A saliency map is computed to characterize each feature and (2) Top-down goal-driven attention, which is related to the game context or goals. For example, users usually put more attention on objects that they can interact with, and less attention on the surrounding environment. Importance factors may vary in different applications, and they need to be identified by the application developer. The importance of each macro-block in a frame is determined using both the saliency map and the importance factors, which are then used to assist with the bit-rate allocation in the encoding process to improve the perceived quality for a specific bandwidth requirement. Their work is focused more on computer vision techniques while the approach we propose uses more graphics information. Furthermore, the fact that the importance factors need to be decided by a human for every application restricts the application of GAM on a wider scale.

Wang and Dey [38] propose a rendering adaptation technique for mobile cloud gaming. The work adjusts the communication and computation cost to satisfy given constraints by changing a set of rendering parameters, including color depth, view distance, texture detail, environmental detail, and frame rate. They consider more rendering parameters than we do in our proposed work. However, the approach is not as generic as ours since not all of these parameters are used in all 3D applications. Besides, an off-line pre-processing step is required to characterize how these parameters affect the communication and computation cost. Another major difference to our approach is the actual outcome perceived by the users. In their work, changing the rendering parameters does not only affect the communication and computation cost, but also changes significantly how the 3D scene looks, which may not be acceptable or even possible for some applications. In our approach, we aim to keep the final visible result intact so that the users have a more consistent experience, irrespective of the resource constraints.

Nan et al. [31] propose a cloud framework specifically for games where the distortion perceived by the players is minimized by maintaining and synchronizing graphics buffers on both the server and the client. At the beginning of the game, the client buffer is empty and the system works essentially as a pure video-streaming system. In addition to streaming the compressed frames, the server also sends graphics data to the client progressively throughout the session. The graphics data is used to render reference frames on both the server and the client. The bandwidth consumption for streaming the frames can be reduced by choosing the reference frame with lowest residual error. The paper formulates and solves an optimization problem where available bandwidth is allocated between the compressed frame and the graphics data to minimize visual distortion. Although they also use progressive meshes in their framework, the approach and optimization goal are different from ours. In their system, the graphics data is sent by the server at run time; thus, the client does not store any data locally nor does the client execute any game logic locally. It basically assumes a 
lightweight client, whereas we allow the end device to determine how lightweight it wants to be. We claim that by using some resources on the client, our system will be able to achieve several important advantages. Storing graphics data locally not only reduces the bandwidth usage but also improves the visual quality especially during scene changes since the application does not need to wait for the graphics data to arrive in the buffer. Executing the application locally requires more computing power on the client, but it allows our method to reduce or even eliminate interaction latency.

Chuah and Cheung [8] propose a layered coding technique to reduce the bandwidth requirement for mobile cloud services such as gaming. The client renders the base layer of the scene by modifying several rendering parameters, including the number of polygons, illumination, and texture mapping. The server compresses and sends the residue, or enhancement layer, to the client for it to improve the final image quality. Their idea is very similar to ours. However, their work does not tackle the practical issue of generating the 3D content for the base layer in real-time. We propose to use progressive meshes to support dynamic generation of graphics data with different levels of detail. Therefore, our system does not only take into consideration the variety in capabilities of clients' devices, but also has the ability to adapt to the ever-changing network conditions to which end users are subjected.

Hemmati et al. [15] adapt the number of objects rendered on the server to reduce the bandwidth requirement. The proposed system monitors the user's actions and prioritizes all of the objects based on their importance given the user's current activity. Depending on the client device's capability, the server renders only a number of the most important objects and streams the resulting frames as a video to the client. Similar to [8], the importance of the objects is decided by the application designers who understand the context of the applications. Thus, it shares the same disadvantages. The quality of the solution depends largely on the designers' ability to identify important objects. It therefore makes it very hard to apply to all existing applications that suffer from the same issues. In addition, simplification by removing unimportant objects is not always possible, especially when multiple objects in the scene are interacting with each other. This approach makes it very hard to fine-tune the system to meet all different performance constraints.

Shi et al. [36] make use of depth information and 3D image warping technique to improve the bit-rate-distortion ratio. The main idea of reducing the bit rate by utilizing low-entropy residue images is similar to many other studies including our approach. However, the residue is calculated by comparing the current frame with a previous frame, instead of comparing frames with different levels of detail. In order to minimize the entropy of the residue image, each frame is transmitted with the viewpoint information so that the frame can be warped to another viewpoint to match a new frame. While making use of the already transmitted data to avoid the need of sending too much additional data is a great idea, there are several fundamental problems. First, the warped image will miss some information due to exposure and sampling. The authors try to solve this problem via double warping, where a previous frame and a predicted future frame are used together to generate a warped frame with the current viewpoint. This method requires a good prediction of change in the user viewpoint. Otherwise, it can result in using even more bandwidth than simply streaming 3D graphics data. A more important problem is related to moving objects. If there are reasonable number of moving objects in the game scene, the warped image will differ from the real image significantly since the image warping technique does not consider the translation of all other objects. Due to these problems, this approach is mostly suited for applications such as remote virtual environment walk-throughs, where most objects are fixed and the movement of the viewpoint is fairly predictable - the path that a user will take through the space is predefined.

Crassin et al. [10] offload computation of three different view-independent indirect lighting algorithms, path-traced irradiance maps, photon mapping, and cone-traced voxels, to the cloud. 


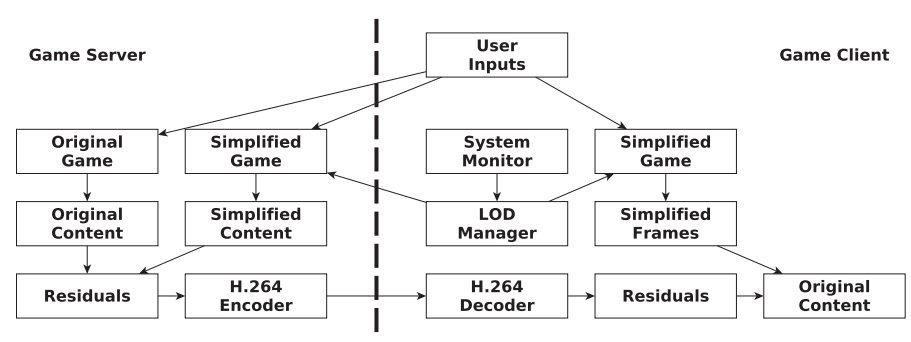

Fig. 1. System model.

For each algorithm, they decide a sweet spot in the rendering pipeline to divide the workload so the network bandwidth requirement is minimized. Since the result is view-independent, this approach can be very useful for multiplayer games. Eu et al. [13] developed a set of tools for game developers to make their games support progressive file streaming more easily.

\section{SYSTEM OVERVIEW}

The main goal of our framework is to reduce the network bandwidth requirement and the endto-end latency between the server and the client while accommodating the hardware resources available at the client. The basic idea is to utilize the available computing capacity on the client machine to generate close-to-original rendering results, thereby only the residual frames are required to be transferred from the server to restore the original visual quality. The system is designed to (1) support a wide variety of client devices with different rendering capability and (2) be flexible, i.e., adjust dynamically to processing loads and network conditions.

Figure 1 provides an overview of the system architecture. A system monitor runs on the client to keep track of the current GPU load and bandwidth usage. The Level of Detail (LOD) manager uses the collected information to decide on a desirable LOD that the environment should display and dynamically changes the 3D models used in the application to meet that requirement. The server runs two application instances, one with the LOD decided by the LOD manager, the other with the original or highest possible LOD. A residual frame is generated by comparing the rendering results of the two versions for each frame. The server encodes the residual frames using an H.264 encoder and sends the compressed data to the client. The decoded residuals are added to the corresponding frames rendered by the client to recover the original quality.

To get the best bandwidth savings, the system requires that all environment instances running on both machines be perfectly synchronized. In other words, the states of each instance need to be consistent when rendering each frame. For now, we use a simple lockstep synchronization to make sure all environment instances read the same sequence of user inputs and execute the same set of state updating commands between any pair of consecutive frames. This means the proposed solution suffers a network latency of a round-trip delay. Latency is a very important issue, in particular for some classes of interactive applications. The initial emphasis in our work is on bandwidth savings for cloud services, and the solution we present below works fine for applications that do not have stringent latency requirements. We discuss the issue of latency in Section 5.

In our experimental system, both the server and the client have the original 3D models, including the mesh information, textures, etc., stored locally before the application starts, so there is no bandwidth consumption for transferring the data. When a new base LOD is agreed upon, both sides run the mesh simplification algorithm and generate the new models independently. The base 
LOD of a model is chosen based upon the availability of resources. Lower-detail base models result in residuals with higher entropy which require more bandwidth but demand lower computation costs at the client. Vice-versa, higher detail models will translate to lower bandwidth requirements, have less residuals, but result in a higher workload at the client. In the next section, we discuss these tradeoffs.

An alternative approach for client devices that lack storage capacity is to transmit the base model information in real time. When a new LOD is chosen by both sides, it asks the server to generate and transfer the new models. While saving storage space, this does require extra network bandwidth for the data transmission and an extra delay will be incurred before the new models are ready to be used on the client. These costs are not considered in our initial investigations of the viability of our approach, since we believe the change in LOD of the base model should not happen too frequently. Other possible approaches are addressed in Section 6 when we discuss future work.

\section{BANDWIDTH REDUCTION}

\subsection{Collaborative Rendering}

In our proposed approach, we make use of the graphics processing capability of the client to reduce the network bandwidth requirement by means of collaborative rendering. In our collaborative rendering scheme, the client renders each frame with a lower LOD and uses the difference image calculated by the server to reconstruct the original high-quality frame. By using delta encoding, the deltas, which are the difference images in our case, can be better compressed if the lower quality frames are close enough in detail to the original.

To calculate the difference image for each frame, we adopt the solution proposed by Levoy [23]. The server first renders both the high- and low-quality frames, which are represented as 24-bit RGB pixels. The difference image is calculated pixel-by-pixel using the formula $D=127+(H-L) / 2$, where $(D, H, L)$ are the pixels in the difference-image, high-quality, and low-quality frames, respectively. As one might notice, this method has the effect of losing one bit of information. However, as our experimental results show, the visual quality of the final outcome is still superior to pure visual streaming in most cases. The difference images are then encoded and sent to the client. After decoding, the client adds the difference image to the low-quality frame rendered locally to reconstruct the original high-quality frame. Since H.264 is lossy, the pixel values of the reconstructed frame may not be in the range $0-255$. We ensure the values are valid by applying the formula $H=\operatorname{Max}(\operatorname{Min}(2 *(D-127)+L, 255), 0)$ to each pixel.

There are several challenges when using the collaborative rendering mechanism. The first issue concerns the interaction latency, that is impacted by network and processing delay. Our method incurs one round-trip network delay, similar to video-streaming-based cloud gaming, if there is no data loss. Assume the application starts with an initial state $S_{0}$. In each loop, it reads a set of user inputs $I_{i}$ to update the environment state from $S_{i-1}$ to $S_{i}$. To generate correct difference images, $S_{i}$ has to be consistent on the server and the client for all $i$. As a result, the server needs to wait for the client to transmit $I_{i}$ before rendering the high- and low-quality frames. The client then has to wait for the difference image from the server to reconstruct the high-quality frame. Since collaborative rendering requires the server to perfectly replay the game instance of the client, the user input has to be synchronized and cannot be lost. We therefore use TCP for transmitting the user input to provide a reliable channel. Several enhancements to TCP have been proposed [33] to reduce the latency incurred by the retransmissions for "thin" streams, such as gaming traffic. The processing delay is expected to be longer due to the additional computation required at the client that consists of rendering, frame comparison and reconstruction. The overall added cost in delay will be discussed in Section 4.3.2. 


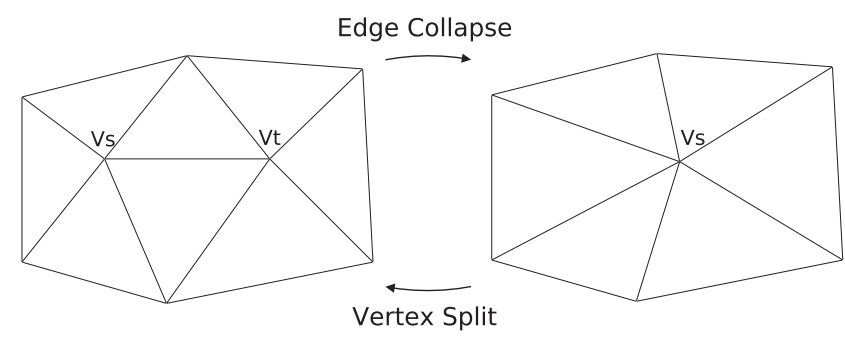

Fig. 2. Mesh collapse and mesh split.

The second issue is related to network bandwidth utilization. There are two key targets to achieving better bandwidth savings. The first is to generate difference images that are more compressible. It is obvious that the more similar the low-quality frame is to the high-quality frame, the easier it is to compress the difference image. In our system, we dynamically adjust the LODs of the environment models according to the client's computing capacity by using progressive meshes, which will be discussed in Section 4.2. The second is the compression method that we use. Developing a dedicated compression method for the difference images is beyond the scope of this article. We chose instead to encode the difference images as H.264 video since the frames share similar spatial-temporal correlation. Despite the fact that the video coding standard H.264 is not designed specifically for this kind of application, our experimental results, shown in Section 4.3, show that we can achieve decent compression ratios. An advantage of using H.264 is that it is a popular standard and is widely supported on devices.

As shown in [11], synchronizing the two game instances can be a difficult task. We do not address the problem here. Instead, we assume that there are no non-deterministic behaviors in our game logic; therefore, the two game instances will produce the same graphical output in each frame as long as the same sequence of user input is received during each game loop.

\subsection{Dynamic Mesh Simplification and Restoration}

As explained in the previous section, the bandwidth savings of the system depend on the graphics computing capability of the client. At one extreme, using our proposed system, a high-end PC that can run the application locally, will have no need for any network communication with the server and the full detailed model mode will be used. At the other extreme, for a client that does not have the computing power to do any graphics computation, the proposed approach will work as a traditional video streaming-based system. For all devices in between, whose computing capability lies between these two extreme cases, the system will perform in collaborative mode. It also works well when the available computing capacity and bandwidth resources for a client vary over time depending on what other applications are running on the client device. There may also be instances where the developer will not release the application as a standalone and only allow users to use it on the provided cloud servers, or the other extreme, not offer it as a cloud service. Our proposed method can operate in any of those modes.

The flexibility of our approach hinges on using progressive meshes proposed by Hoppe [17] to enable dynamic change of the Level of Detail (LOD), in terms of the number of edges, of the base quality models. The transformation is accomplished by two operations: edge collapse and vertex split. Figure 2 illustrates an example of both operations. As shown in the figure, an edge collapse unifies $v_{s}$ and $v_{t}$ into a single vertex $v_{s}$. The edge connecting the two vertices and the faces associated with it are removed. A vertex split is the direct opposite of an edge collapse. It 
splits a vertex into two vertices and creates a new edge between them, resulting in one or two additional triangles.

To construct the progressive mesh representation for a given mesh, we need to decide on a sequence of edge collapses that simplifies the original mesh until there is no edge to collapse or the number of edges is lower than a certain threshold. For the work reported here, we use the algorithm proposed by Garland and Heckbert [14] to decide the sequence. It is a very popular approach and has been shown to have good results. The effect of using other mesh simplification algorithms will be investigated in future work. For each edge collapse, the attributes of the affected vertices and faces, e.g., the position of the new vertex, are recorded. These records are used in future vertex splits to transform the mesh from a simpler form to a more complex one. It is shown by Hoppe in [17] that the progressive mesh can be space-efficient with carefully designed encoding. However, since speed is more important than space in our application, we did not make any attempt to reduce the space usage. Many mesh simplification algorithms with different tradeoffs between speed and accuracy can be used to construct the progressive mesh. In some applications, the progressive meshes can be constructed during the scene loading phase, in such cases, accuracy is considered more important than time complexity.

In our system, the LOD manager constructs and maintains a progressive mesh for each model loaded into the system. It dynamically decides on an ideal LOD based on the information acquired by the system monitor on available resources, then transforms the meshes using a sequence of edge collapses or vertex splits until they match the target LOD. The higher the LOD used for the locally rendered models, the more accurate it is in approximating the original ones, which results in less network bandwidth for transmitting the difference. However, rendering higher quality models takes more graphics computing power. Therefore, the LOD needs to be carefully selected to achieve acceptable computational cost, with highest bandwidth savings. Although there are many other factors that affect the overall LOD in modern game engines [27], their effects on the required computing resource are usually hard to predict. As a result, we limit our configurable LOD as simply the total number of edges of the 3D model, which has a more predictable relationship with the required computing power, as discussed in Section 4.3.2.

An alternative is to use a set of pre-built models with different LODs, as proposed by Cuervo et al. [11]. Although constructing progressive meshes requires some time and effort, it comes with several important advantages. First of all, progressive meshes provide a continuous sequence of models with different LODs. It makes the system more flexible and can be dynamically fine-tuned to adapt to different combinations of hardware capabilities, usage cases, and network environments. Second, since the progressive meshes are generated automatically as long as the original meshes are accessible, our solution is not limited to applications that have configurable LOD. Third, since the progressive meshes can be generated dynamically, we do not need to store multiple versions of a single model. This is especially important for mobile devices where the storage capacity is extremely limited. One last advantage of progressive meshes is that they naturally support progressive transmission. Although we currently assume the client can access the original models and construct the progressive meshes locally, it is possible to eliminate this storage and computation overhead by transmitting the simplified models on demand from the server. Progressive transmission will turn out to be useful in such situations.

\subsection{Performance}

To measure the performance of our approach, we developed an OpenGL-based application where a player can freely walk around a virtual city. The Default 3D model of the city contains 158,504 faces and 36 texture files. For the work presented in this article, we did not address the issue of updating the texture mapping when the LOD changes, instead, we generated a non-textured model 


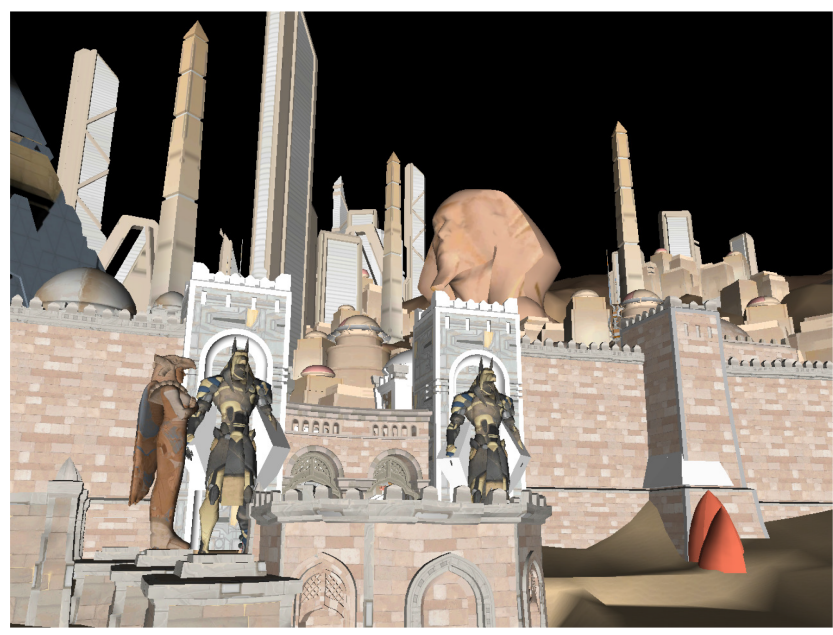

Fig. 3. A sample empirical game scene: Overwatch.

which replaces the textured content with the average color of the texture for every face. Both the textured and non-textured models are tested and analyzed to investigate the effect of our scheme. In order to investigate the effect of model complexity on the performance of our scheme, we tested a simpler model (Simple Model), which has 15,228 faces and 16 texture files, and a more complex model (Complex Model), which has 340,409 faces and 248 texture files. For the purpose of proving that our approach can be applied to a real 3D game world, we also created a 3D model (Empirical Model) based on a scene in a popular online first-person shooter game, Overwatch, developed by Blizzard Entertainment. As shown in Figure 3, the Empirical Model has very similar features to the game scene "Temple of Anubis" in Overwatch and has 926,034 faces and 90 texture files. We define the base LOD as the number of edges in the base mesh divided by the number of edges in the original mesh. Figure 4 shows the Default Model using different base LOD values.

For a fair comparison across all the models, we scaled them to approximately the same virtual area. We recorded six types of movement commonly encountered in most 3D games: (1) moving forward for 360 frames, (2) moving backward for 360 frames, (3) shifting to the left for 360 frames, (4) shifting to the right for 360 frames, (5) turning left 360 degrees in 720 frames, and (6) turning right 360 degrees in 720 frames. Each movement sequence is replayed with base LOD set to $0 \%$, $10 \%, 20 \%, \ldots 100 \%$, and all relevant information is saved for analysis. We average the performance of the six types of movement to get an overall performance for movement through the space. In the case of a base LOD of $0 \%$, we stream the original frames instead of difference images, i.e., the approach reverts to traditional video streaming. At the other extreme, for a base LOD value of $100 \%$, the approach reverts to operation as a standalone device.

The environment is set up on a server that runs on a 64-bit Windows 7 PC. The client is run on a Lenovo X1 Carbon laptop running the Ubuntu 16.04 operating system. Both machines are connected to the same local area network. Color images are transformed to YUV420 format before encoding. We use libavcodec version 57.107.100 and libx264 encoder. As for the H.264 configuration, we use a frame rate of 30FPS, group of picture (GOP) of 10, and a target output bit rate of $8 \mathrm{Mbps}$. To minimize the latency, we use preset ultrafast and tune zerolatency. Since the zerolatency setting basically disables look-ahead and the use of b-frames, it will be harder for the encoder to maintain that target and will result occasionally in output bit rates larger than $8 \mathrm{Mbps}$. The prototype application is rendered and encoded at $1280 \times 960$ pixel resolution. 


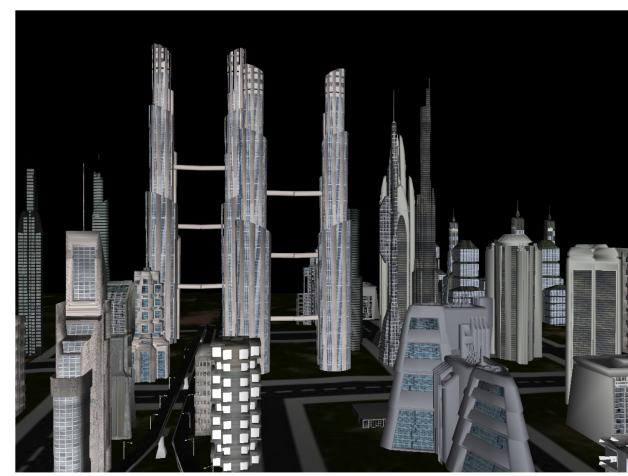

Original

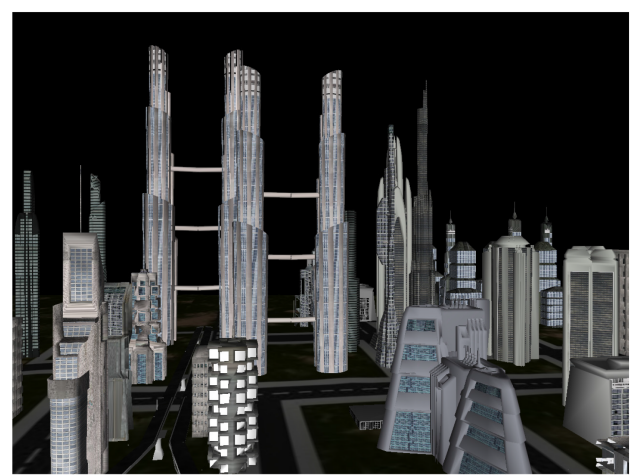

$60 \%$

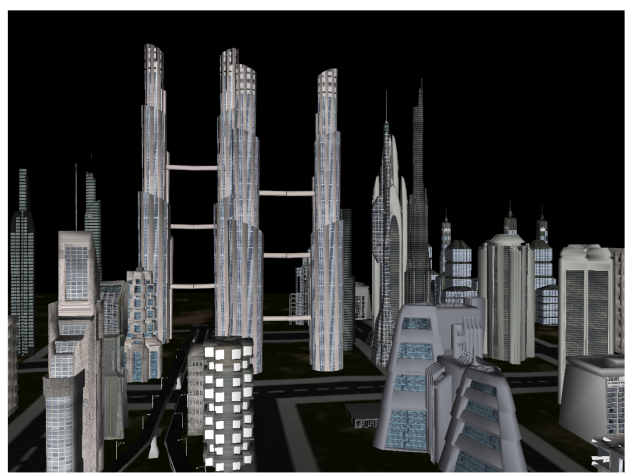

$90 \%$

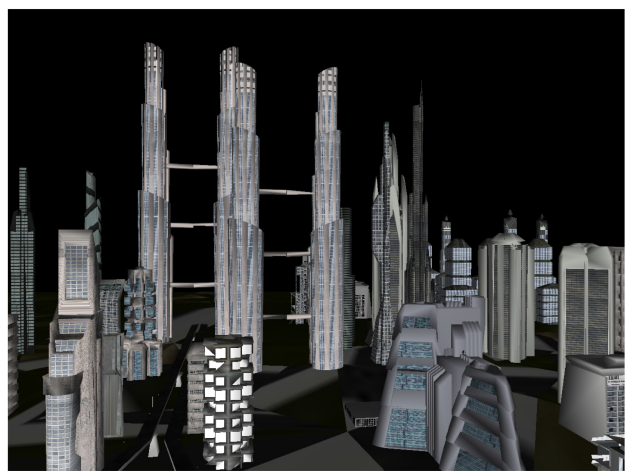

$30 \%$

Fig. 4. Rendering results of Default Model with different LODs.

Our comparison measurements focused on three performance metrics: network bandwidth, rendering delay, and visual quality. For network bandwidth, we are only interested in the encoded difference images sent from the server to the client, since the control messages sent from the client to the server are very small and can be virtually ignored in comparison to the video stream bit rates. The rendering delay is the time the client uses to render the base model in each frame. We use it as an indicator of the graphics computing workload on the client hardware. For visual quality, we calculate the PSNR from the original image rendered by the server and the reconstructed image generated by the client for each frame. Since we are using a lossy compression algorithm (H.264) to encode the image differences, there is a tradeoff between compression ratio and image quality. We need to be aware of how much quality is sacrificed for bandwidth savings. In the remainder of this section, we will show how the base LOD values affect these three performance metrics.

4.3.1 Network Bandwidth. Figure 5 shows how the base LOD values affect the bandwidth usage for the Default Model. The average size of the encoded difference images is very small when the base LOD is higher than $70 \%$, then it starts to grow faster as the LOD drops. When the base LOD is lower than $50 \%$, the bandwidth savings become insignificant for the textured model.

We observed an interesting artifact for low LOD values, some buildings disappear completely, resulting in the objects behind them becoming visible, which may increase the size of the encoded difference image dramatically. A potential solution is to use a view-dependent progressive mesh that tries to keep faces that are closer to the viewpoint intact. However, view-dependent 


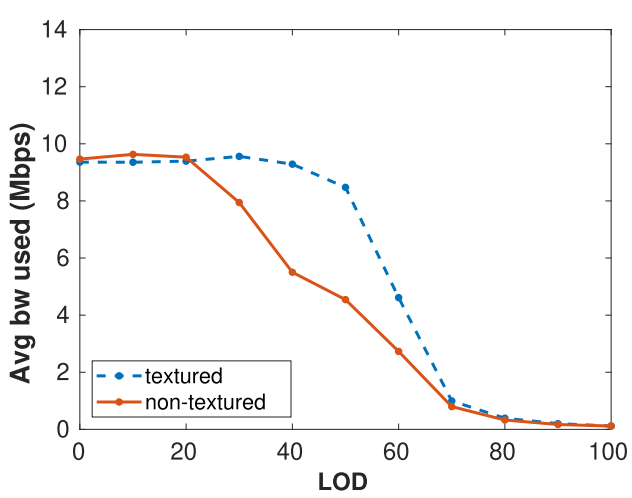

Fig. 5. Bandwidth comparison (Default Model).

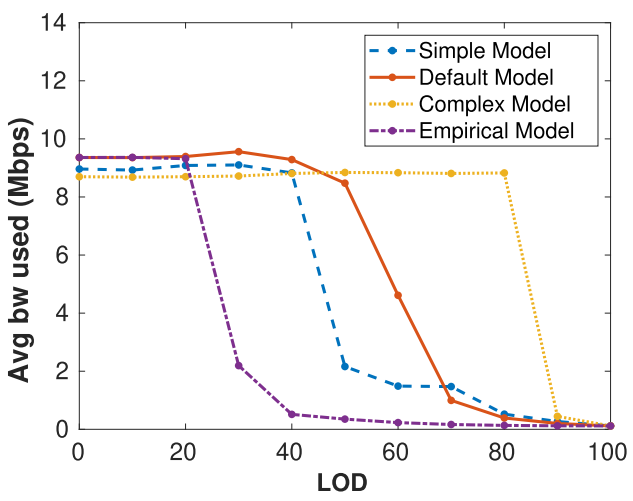

Fig. 6. Bandwidth comparison (all textured models).

progressive meshes need to be updated whenever the user changes the viewpoint, which happens frequently in many of these immersive applications. Finding a way to reduce the bandwidth usage in low base LOD with acceptable extra effort is an important issue that we will address in Section 6 under future research.

Another reason that there is almost no bandwidth reduction for the textured model when the base LOD value is low is the lack of a good mechanism to update the texture mapping when LOD changes in the current progressive mesh implementation. When the mesh is simplified, several faces may be merged into a larger one. To more accurately approximate the original mesh, the texture mapping of the remaining faces should be updated accordingly. Otherwise, the texture will be stretched and thereby introduce a significant difference in the two frames. When the base LOD is decreased to a fairly low value, the faces that introduce large differences may disappear altogether, which will actually reduce the complexity in the difference images. This effect may explain the irregular trend of bandwidth usage when the base LOD is between $0 \%$ and $50 \%$.

Figure 6 shows the impact of the model complexity on the bandwidth usage when textured models are used. When the Simple Model is used, a lower LOD is sufficient to represent the original model and the bandwidth usage drops earlier than for the Default Model. For the Complex Model, it becomes very hard to get any bandwidth reduction when the LOD is lower than $90 \%$. That is because a large number of faces across the whole game world will be merged, even with a slight change in LOD, thus the inaccurate texture mapping has a larger impact on performance. To our surprise, we actually see the highest bandwidth savings for the Empirical Model, we start to see significant bandwidth drop when the LOD is higher than $30 \%$. We believe one possible reason is that the scene is a city in a desert and it is more monochromatic compared to other models. We also found that beside having more complex 3D models in the scene, the Empirical Model has more objects placed close to each other all around the scene. That means even if some of the objects are totally removed from the scene by the mesh simplification algorithm, the scene looks fairly similar to the original one.

4.3.2 Rendering Delay. The advantage of rendering lower LOD models locally on a client is to lower the computational workload associated with the graphics. We use the time it takes to render a frame to quantify the computing power used by the graphics hardware. As most applications are configured to run using a specific frame rate, the shorter time it takes to render a single frame, the better it will be for a lower powered machine to achieve the required frame rate. Figure 7 illustrates a linear relationship between the rendering time per frame and the base LOD for the Default Model, 


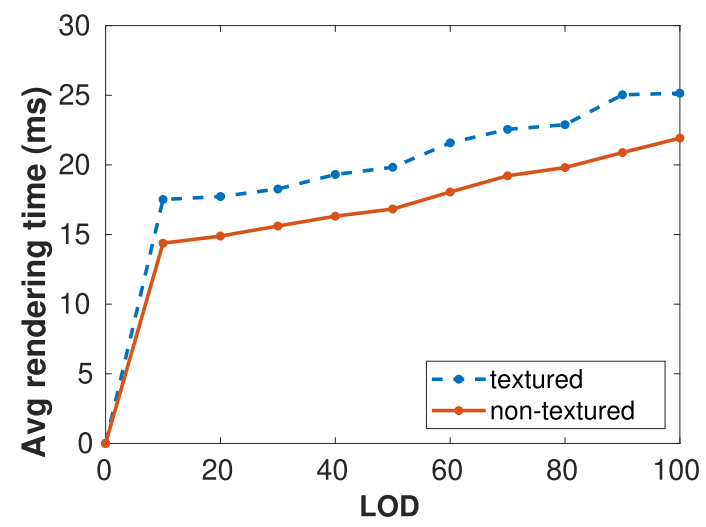

Fig. 7. Rendering delay comparison (Default Model).
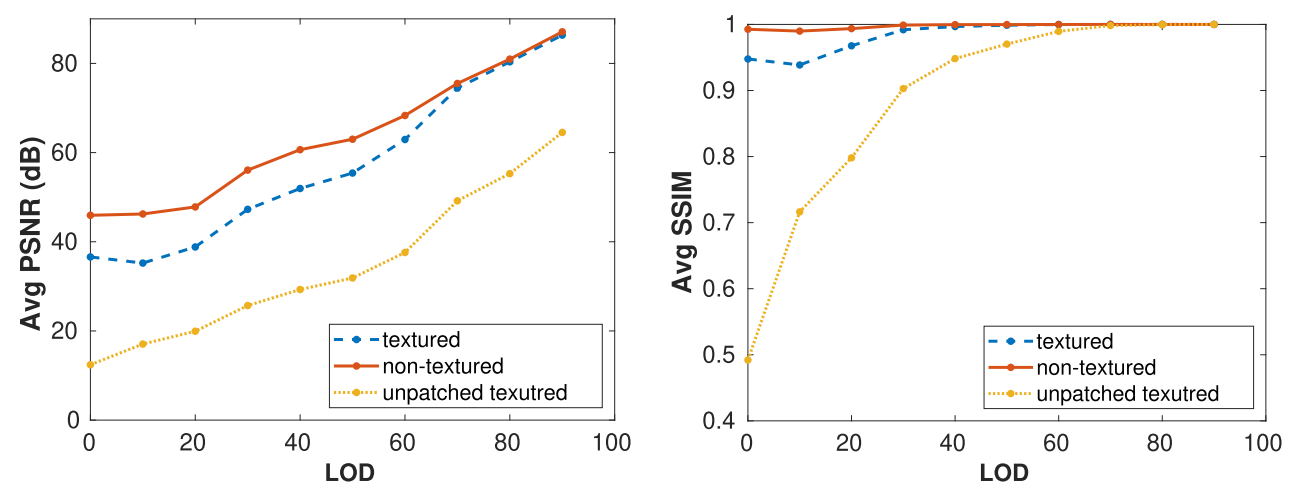

Fig. 8. Visual quality comparison (Default Models).

which proves that using progressive meshes allows us to flexibly adjust the workload requirement based on the available graphics computing power.

4.3.3 Visual Quality. The visual quality is impacted in our system for two reasons. First, we use H.264 to encode the difference images. The H.264 standard was designed to encode a specific type of data, and our data is slightly different. Specifically, it is already a residual when fed to the encoder and has different value distribution, so the final result may not be optimal. The issue can be addressed by using user-specified quantization tables to make it more suitable to the data that is being processed. The challenge lies in creating these quantization tables for this type of data. Second, as was mentioned in Section 4.1, the difference images lose one bit of information for every pixel before encoding, this could very possibly impact the quality further. One possible solution is to compute $D$ as float or 16-bit value and down-sample after quantization, it would add to the computation overhead at the server, but not by much compared to the other computations, such as rendering.

Surprisingly, in spite of the less than ideal residual coding method, our experimental results show that the system is capable of providing acceptable visual quality with significant bandwidth gains. As shown in Figure 8, all collaborative rendering cases that use a base LOD value higher than $10 \%$, generate better visual quality than traditional video streaming in terms of average PSNR. The system achieves a PSNR of about 87.5 when the LOD is $90 \%$, regardless whether textured or non-textured models are used. The PSNR value drops gradually to about 45.5 and 35 , when 


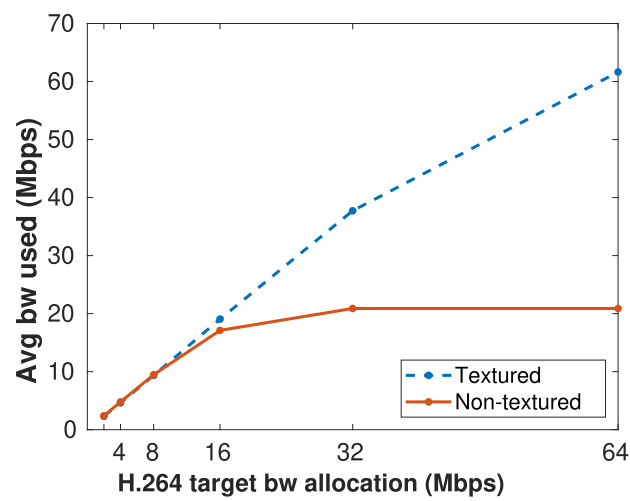

(a)

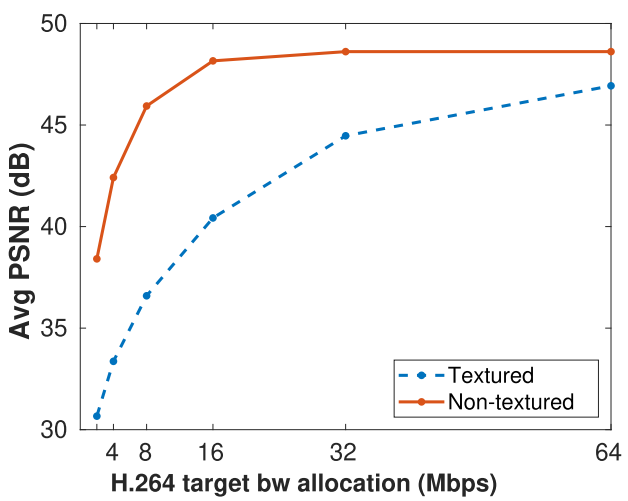

(b)

Fig. 9. Effect of H.264 target bandwidth on video streaming (a) bit rate, (b) quality.
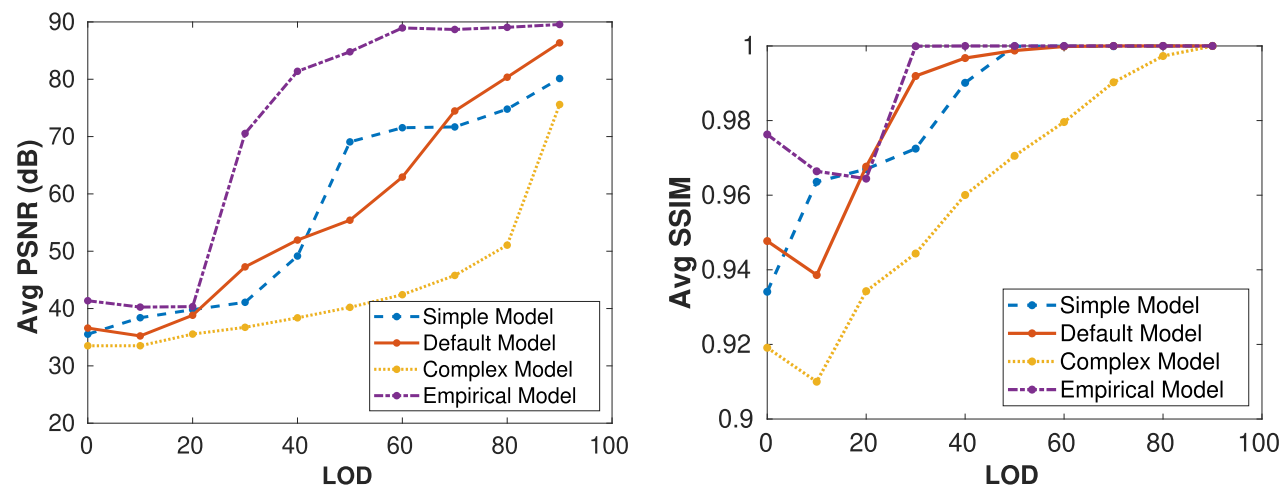

Fig. 10. Visual quality comparison (all textured models).

non-textured models and textured models are used, respectively. In terms of average SSIM, the measurement is near 1, which means least distortion, with higher base LODs. The performance starts to degrade rapidly as the base LOD decreases below $30 \%$. When the non-textured model is used, the lowest average SSIM is about 0.99 , which is still an acceptable quality. The performance is worse when we use the textured model, where the average SSIM goes down to as low as 0.94 . However, the relative performance is not that much lower than traditional video streaming, since the more complex frames generated by the textured model are harder to encode efficiently even for traditional video streaming. To show the impact of the server assistance in the rendering process, the visual quality of the textured model when the residue is not used are also presented in the figure. As shown in Figure 9, it takes at least $16 \mathrm{Mbps}$ for traditional video streaming to achieve a PSNR of 40 or higher. That is a very high bandwidth cost to pay to achieve that quality level.

When comparing models with different complexity, as shown in Figure 10, we found that the visual quality improves rapidly for the Simple Model. Both the PSNR and SSIM values are better than video streaming even when the LOD is only $10 \%$. For the case of the Complex Model, the performance improves at a slower rate as the LOD gets higher. In terms of PSNR, the value can only achieve a value of 50 for a base LOD of $80 \%$ and higher. A similar pattern can be observed for SSIM, where the value only reaches 0.99 when the base LOD is higher than $80 \%$. Once again we see that the best performance occurs when the Empirical Model is used, where the PSNR and SSIM reach 70 and 1 accordingly, for an LOD that is only $30 \%$. 

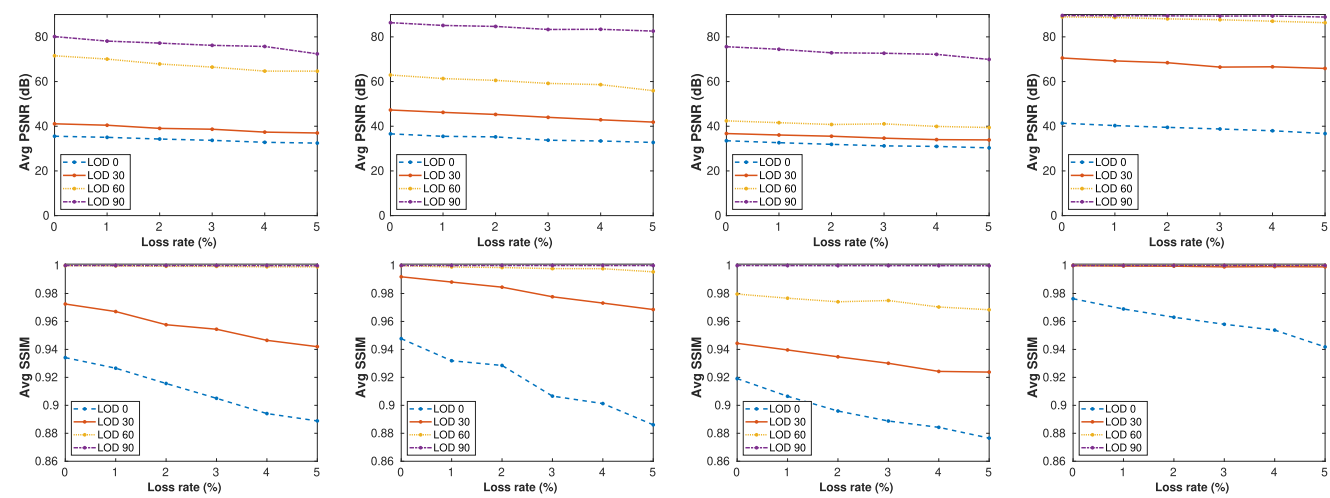

Simple Model

Complex Model

Empirical Model

Fig. 11. Visual quality vs. loss rate (all textured models).

With unstable network connections, packet loss can have a huge impact on the performance of a cloud gaming system. In general, since end user input (action commands) does not require much bandwidth, the loss problem can be mitigated by using enhanced TCP [33] as discussed earlier in Section 4.1. For the streaming scenario, the loss of data from the server to the user can be recovered by the H.264 video encoder, where several post-processing methods exist to mask lost data.

However, we expect that the packet loss impact will be smaller for the collaborative rendering scenario proposed here, as a portion of the data is generated locally on the client. To verify that, we designed a simulation experiment that drops image frames randomly with loss rate ranging from $0 \%$ to $5 \%$. The result is shown in Figure 11. In terms of PSNR, the impact of data loss is similar no matter what base LOD model is used. Nevertheless, the advantage of rendering higher LOD models on the client is substantial in terms of SSIM. For pure video streaming (LOD 0), the SSIM value decreases sharply for all model complexities as the loss rate increases from $0 \%$ to $5 \%$, while the performance is almost unaffected when the base LOD is $60 \%$ or higher.

\section{REDUCING INTERACTION LATENCY}

Interaction latency is an important performance metric for interactive cloud-based services. User studies show that the maximum tolerable delay for online interaction is around $100 \mathrm{~ms}$ to $150 \mathrm{~ms}$ [ 4 , $12,34]$. High interaction latency not only damages users' subjective impression of the service, but can also affect their objective performance. It is shown in [21] that it is possible to trade off some resources to reduce latency. But different types of applications generally have different latency requirements. In general, slower-paced environments (chatting, walk through), usually have a higher tolerance for interaction delay than faster-paced ones (action, drive through). Our aim therefore is to design a mechanism where the resource tradeoffs can be modified more flexibly.

In a naive lock-step system, the client always waits until it gets the residue for a certain frame from the server before it displays the patched frame to the user, as is shown in Figure 12. The interaction latency in this implementation depends on the network round-trip time between the server and the client, which is unpredictable and usually not acceptable for highly interactive applications. Since the client already renders a lower LOD version of the frame locally, it is possible to reduce the interaction latency by sacrificing some visual quality.

\subsection{D Image Warping}

The main idea of our approach is to make use of previous residual frames that the client has already received. In such a system, the client sets a fixed delay period for each frame to be displayed. At 


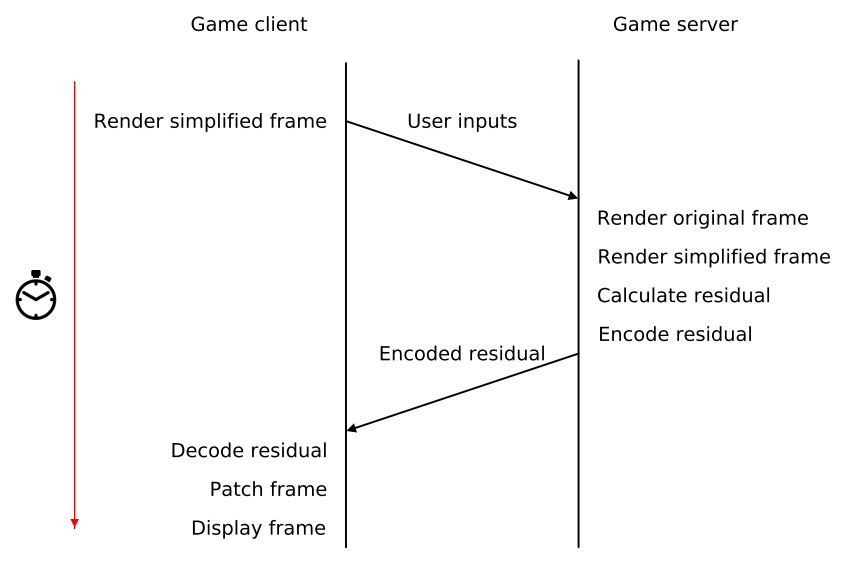

Fig. 12. Lock-step approach.
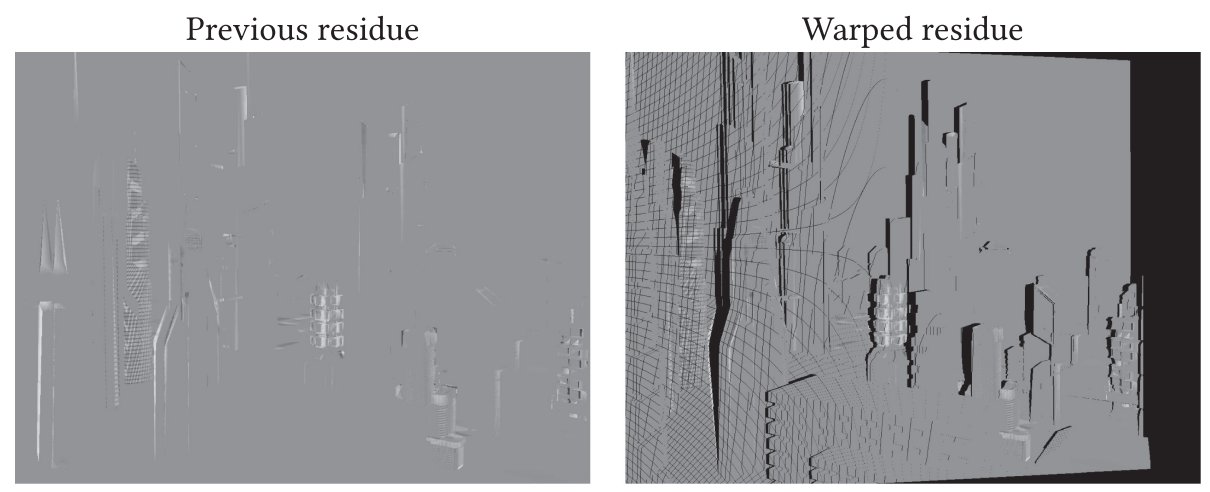

Fig. 13. 3D image warping.

the time a frame is due to be displayed, the client first checks if it has received the residue for this frame. In the case where the residue is ready on the client, it patches the frame and displays it as normal and there will be no extra degradation to the image quality. When the residual frame has not been received, the client uses the last residue it has received and buffered for patching the current frame. The viewpoint of the previous residue is expected to be very close to the current viewpoint, but they are usually not exactly the same.

In order to make the previous residue match up better with the current frame, we use a $3 D$ image warping technique [26]. For 3D image warping to work well, we need extra data about the viewpoint and depth information for each frame. The viewpoint can be retrieved locally by the client. But since the client runs the game with lower level-of-detail objects, the depth information it generates locally is not enough for accurate 3D warping. Instead, we calculate the depth of each pixel on the server using the formula $Z=\operatorname{Min}\left(Z_{\text {high }}, Z_{\text {low }}\right)$, where $Z_{\text {high }}$ and $Z_{\text {low }}$ denote the depth values of the high-quality and low-quality frames. This information will then be transmitted to the client, where the 3D warping actually takes place.

In Figure 13, we show an example of how it works. Due to occlusion exposure or insufficient sampling, the information of some pixels in the warped image will be missing. Obviously, when the viewpoints of the two frames are close, less information will be missing. Thus, there is a tradeoff between latency and visual quality. If the client can wait a longer period (lower interactivity), 


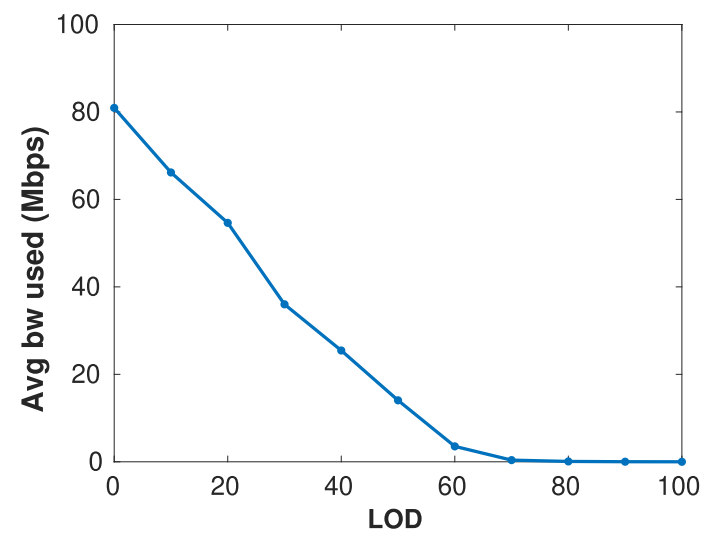

Fig. 14. Bandwidth requirement for depth images.

it might receive a residue that matches up with the current frame better and less information will be missing. In our proposed system therefore, the target latency is a configurable variable and can be changed in real-time. The tradeoff as always is image distortion. There are two sources of disocclusion: (1) areas previously occluded by objects and (2) areas around image boundary. Many methods have been proposed to address the first type of disocclusion. Most of the existing work focuses more on improving the quality of the recovered image. Reducing the computational cost is arguably more important than recovery quality in our application for a couple reasons. First, since the image warping and hole filling have to be done on the client side, it will increase the workload on the device that normally will have a lower computing capability. Second, the missing information is just the residue information. That means that we can most likely get a reasonably accurate value even without recovering the residue. Therefore, our current prototype system uses a low-cost method proposed in [44] instead of other more complicated methods. It addresses the problem by asymmetrically smoothing out the depth discontinuities before the warping. This method is ideal for our application because the smoothing process is independent of the target viewpoint and can thus be done on the server.

In contrast, filling disocclusion areas around an image boundary is hard in most usage cases but can be addressed rather easily in our application. Since we actually have information of the disoccluded areas, a straight-forward method is to have the rendered viewport a factor larger than the displayed area. In other words, the system maintains information around the visible boundary so it may be used after warping.

The resulting 16-bit depth frame is compressed using the lossless compression method proposed in [41] to avoid having one more factor to analyse. Notice that if the color of a pixel is the same on the high- and low-quality frames, the residue is zero and has no effect on the patched image no matter where the pixel is warped to. In this case, we set the depth value of the pixel to zero, which is an invalid value and will be ignored in our warping process. This trick helps us to reduce the size of the compressed depth frame especially when the base LOD is high. The bandwidth requirement for the compressed frames is shown in Figure 14. Although the compression ratio is good for a lossless compression, the bandwidth usage is fairly high when the base LOD is lower than $50 \%$. We are investigating lossy compression methods that will bring the bit rate down. Some H.264 or H.265 implementations support lossy compression of 16-bit depth images. Their impact on the final visual quality is left for future study.

One problem of our latency reducing approach is that it requires considerable computation overhead and may not be applicable for all devices. In our prototype system, the whole pipeline 

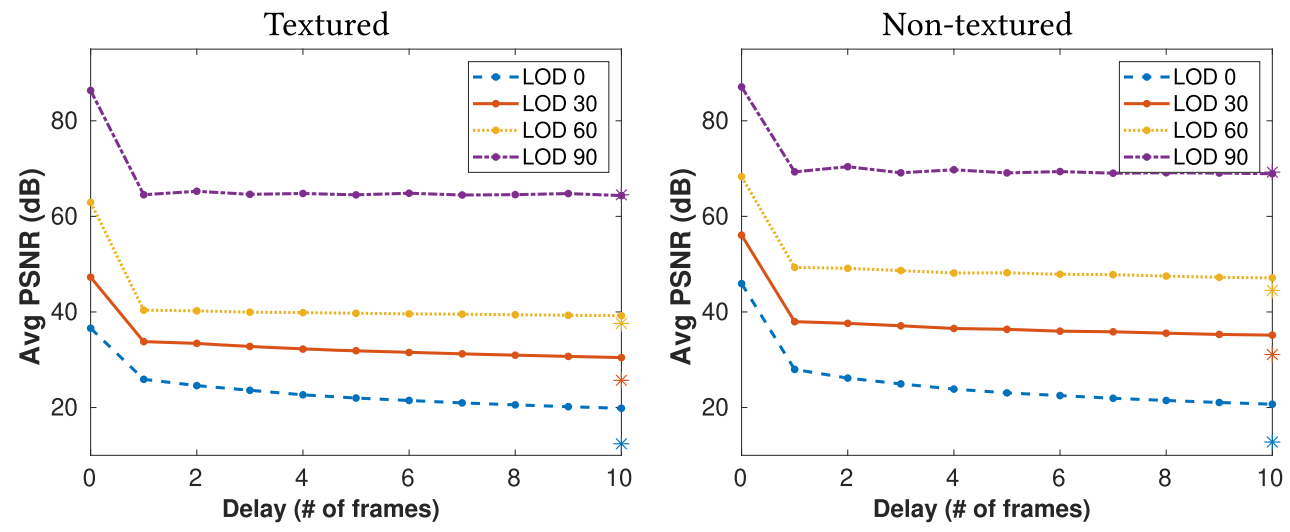

Fig. 15. PSNR vs. delay (Default Model).
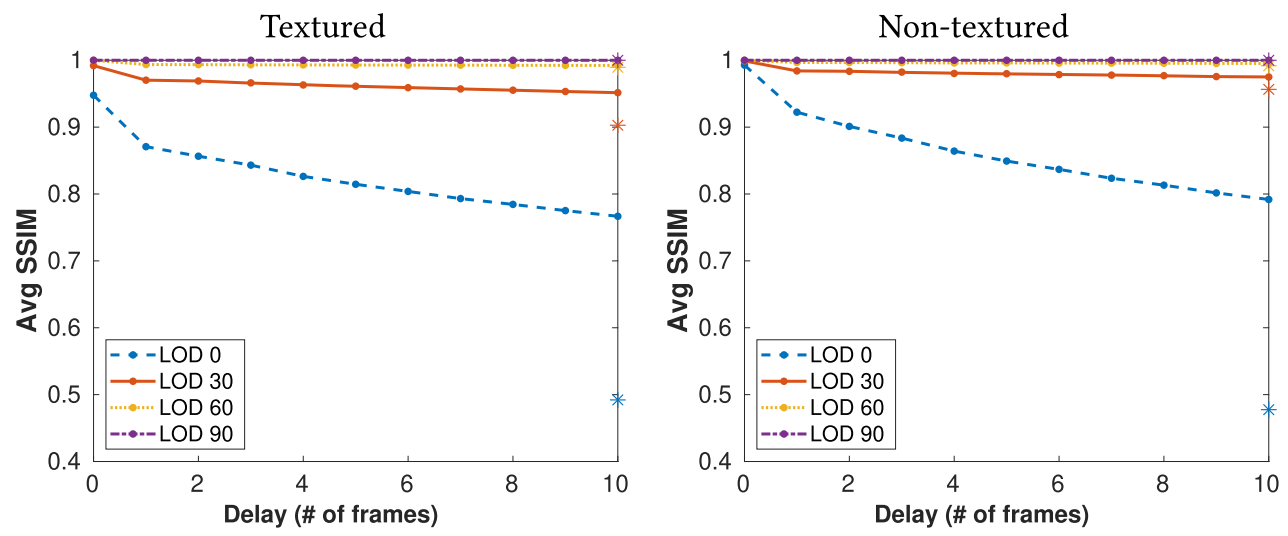

Fig. 16. SSIM vs. delay (Default Model).

except the encoding/decoding process is done with multi-pass shaders and can achieve at least $30 \mathrm{fps}$ on the machines used for our experiments. However, further research is required to identify classes of client devices for which this may not be true.

\subsection{Performance}

To understand the performance of our latency reduction approach, we conduct an offline experiment that changes the interaction latency, in terms of number of frames, and measures the visual quality of the output video. For example, when the delay is set to $N$, the system always uses the residual frame it received $N$ frames earlier to patch the current frame for display. We assume that the original depth information for each residual frame is available on the client. All of the other settings are kept the same as for the previous experiment. The results of the PSNR and SSIM measurement of different LODs are shown in Figure 15 and Figure 16, respectively. To show the limit of the approach, we ran it for the case where the residual frame is not received/used at all. Those results are marked by the asterisks in the figures (shown on the 10 tick marker on the x-axis).

When the delay is 0 , the residue of the current frame is available on the client and can be used for patching directly without being warped, thus there will be no distortion due to the 3D image warping procedure. When the delay is larger than 0 , image warping is required and a significant performance drop can be seen in the figures, regardless whether textured or non-textured models 

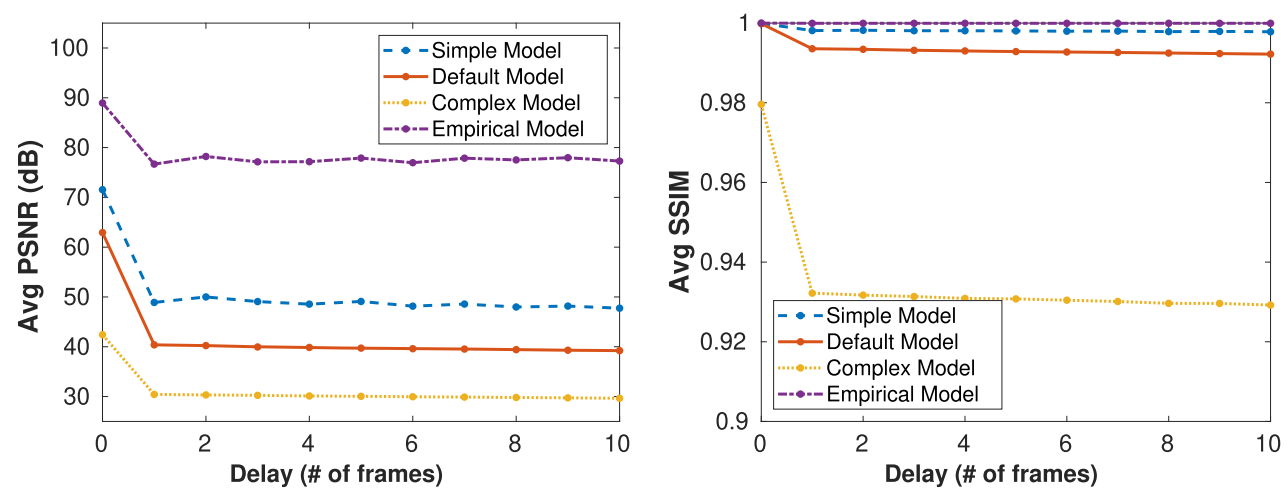

Fig. 17. Visual quality vs. delay (all textured models with LOD of $60 \%$ ).

are used. As the delay increases, the difference in the viewpoints of the current frame and the residual frame becomes larger. As a result, the image warping process causes more information loss and the image quality decreases gradually.

When comparing the results of different LODs, we found that the delay affects the image quality more significantly when lower quality models are used. We believe the discrepancy can be explained by the amount of information within the residual frames. When higher LOD models are available, the locally rendered frames are very close to the original ones, and very little meaningful information is provided by the residual frames. In such a case, the benefit can be easily diminished by the distortion caused by the image warping. On the other hand, if lower LOD models are used, the client depends largely on the residual frames to display the image with reasonable quality. Thus, even distorted residual frames can provide large amounts of useful information that helps improve the image quality. Nevertheless, using the warped residue always has positive impact on the image quality in all of our experiment cases. Whether the improvement on image quality is worth the extra computation and communication costs requires further study.

Figure 17 compares the PSNR and SSIM measurement for different model complexities. The base LOD is set to $50 \%$ for each model. In general, the visual quality is better with lower model complexity, due to the fact that less information is required to restore the original model. This is in accord with results shown in Section 4.3.3. Although we found that the SSIM value drops slightly faster as delay increases when the model complexity is higher, the delay generally has similar impact on the visual quality for all models. We therefore believe that our interaction latency reduction method is feasible even with higher model complexity.

Figure 18 and Figure 19 show the bandwidth usage (not including bandwidth used by depth frames) and the resulting image quality when the relative viewport size is set to different values, for Default model with LOD 60\%. 100\% means the original resolution $(1280 \times 960), 110 \%$ means the viewport size is 10 percent larger than the original $(1408 \times 1056)$, etc. From the results in Figure 19 , we see that a $10 \%$ increase in viewport size corresponds to barely a $1 \%$ improvement in quality in terms of PSNR. A similar improvement pattern was noticed when we measured SSIM. From the figure, we also see that a further increase in viewport size shows very little quality improvement. However, we do see a linear increase in bandwidth usage with increase in viewport size as shown in Figure 18. From these plots, one can conclude that more than $10 \%$ increase in viewport size is not worth the extra cost in bandwidth based on these quality measures. However, a user study or a better perceptual video quality assessment method [28-30, 35, 39, 40, 42, 43] might prove otherwise and show markedly improved quality results that warrant the cost. This is left for future work. 


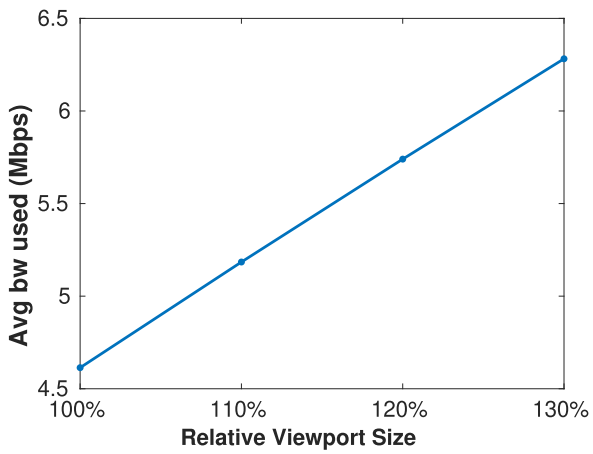

Fig. 18. Effect of viewport size on bandwidth.

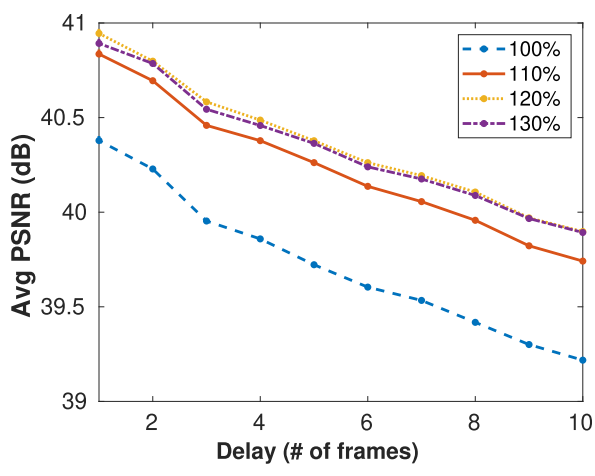

Fig. 19. Effect of viewport size on visual quality.

\section{CONCLUSIONS AND FUTURE WORK}

In this article, we proposed a streaming framework for 3D interactive cloud services such as gaming and virtual environments. By utilizing techniques such as collaborative rendering and progressive meshes, the proposed system dynamically adjusts its resource usage to fit different hardware constraints and performance requirements. The proposed approach presents a flexible solution for providing gaming services to devices with different capabilities and having different network resources. Our experimental results show that rendering time on the client is almost linear to the number of edges in the base model, and that in most cases the system provides better quality than pure video streaming. However, the bandwidth usage can be higher than pure video streaming when less than $50 \%$ of the edges are used in the base model, which may be caused by inaccurate texture mapping when the model is simplified. We plan to implement a better texture mapping algorithm in future work for the progressive meshes in order to lower the entropy in the residue and decrease the bandwidth usage for lower LOD models.

In order to tackle the interaction latency issue, we utilized 3D image warping techniques whereby previously received information is used to improve the image quality of the current frame. With this technique, the delay can be flexibly controlled to better meet a required latency. However, reduction in the interaction latency will come at a cost of image quality. The experimental results show that it is possible to eliminate interaction latency of up to 10 frames, or 333 milliseconds, usually considered a noticeable lag, and still provide an acceptable image quality. However, for that acceptable quality, extra information, such as accurate depth information, needs to be sent from the server for 3D image warping to work, adding to the required bandwidth.

View-dependent progressive meshes is another topic for future work. In our current prototype, the LOD of all environment objects are kept synchronized (all the same). The LOD is calculated as a function of the available resources on the client device. But as different objects may have different importance, it would be better if we can set the LOD of each object separately depending on its importance. There are two types of importance one can consider. One is related to the application context, the other is related to the geological locations of the objects or view-dependent LOD [18, $19,22]$.

In the current version of our system, the residual images are encoded to H.264 video format. Although residual images may have similar temporal and spatial correlations to those of a regular video stream, there are subtle differences between these two types of data: (1) the value distribution is less dispersed in the residual images and (2) the pixel values hold less importance in residual images, especially when higher LOD content is rendered on the client. Thus, existing encoders 
that are designed for regular images may not be able to achieve an optimal compression ratio for residual images. A better approach would be to design a specialized codec, or modify some parts of an existing one, such as the quantization mechanism or the entropy coding, to make it more efficient in compressing residual images.

Moving objects are an important issue for reducing latency. The 3D Image warping technique only considers the change of the viewpoint but not the movement of other objects in the environment. Our plan is to implement a two-layer rendering approach. The first layer includes all stationary objects, and the second layer includes moving objects and some other on-screen information such as menu, and so on. The first layer is patched by the residual image, and the second layer is rendered locally on top of the first layer and is not affected by the residual image. As a result, moving objects need to have higher LOD. The issue of supporting different LODs for different objects will be the focus of future work when we take into consideration both user movement and moving objects.

The use of collaborative rendering and progressive meshes means that our approach has to be intrusive. We therefore believe that the best way to make the proposed framework available is by integrating it with an existing 3D environment engine. While the involvement of the application developers is unavoidable, one of the main design goals is to make the tool as easy to use as possible so developers can easily apply our framework to their applications. With the help of progressive meshes, generating different LOD representations of an object is done automatically. However, as mentioned previously, there are several attributes that can be associated with an object, such as: (1) whether it is a moving or stationary object and (2) the minimum acceptable LOD for the object based on its importance. These attributes will be considered in the LOD management component of our framework. To further help the system improve in quality, we plan to build an interface for the developers to specify requirements on how their system should operate. Available requirements will include desirable frame rate, maximum acceptable latency, available bandwidth, and the like.

\section{REFERENCES}

[1] Agam Shah. 2016. The PC upgrade cycle slows to every five to six years, Intel's CEO says. Retrieved on June 30, 2019 from https://www.pcworld.com/article/3078010/hardware/the-pc-upgrade-cycle-slows-to-every-five-to-sixyears-intels-ceo-says.html.

[2] Amazon Web Services. 2018. Amazon EC2 Pricing. Retrieved on June 30, 2019 from https://aws.amazon.com/ec2/ pricing/.

[3] Hamed Ahmadi, Saman Zad Tootaghaj, Mahmoud Reza Hashemi, and Shervin Shirmohammadi. 2014. A game attention model for efficient bit rate allocation in cloud gaming. Multimedia Systems 20, 5 (01 Oct. 2014), 485-501. DOI : https://doi.org/10.1007/s00530-014-0381-1

[4] Tom Beigbeder, Rory Coughlan, Corey Lusher, John Plunkett, Emmanuel Agu, and Mark Claypool. 2004. The effects of loss and latency on user performance in unreal tournament 2003®. In Proceedings of the 3rd ACM SIGCOMM Workshop on Network and System Support for Games (NetGames'04). ACM, New York, 144-151. DOI : https://doi.org/ $10.1145 / 1016540.1016556$

[5] W. Cai, H. C. B. Chan, X. Wang, and V. C. M. Leung. 2015. Cognitive resource optimization for the decomposed cloud gaming platform. IEEE Transactions on Circuits and Systems for Video Technology 25, 12 (Dec. 2015), $2038-2051$. DOI : https://doi.org/10.1109/TCSVT.2015.2450171

[6] D. Chen and M. El-Zarki. 2017. Impact of information buffering on a flexible cloud gaming system. In Proceedings of the 15th Annual Workshop on Network and Systems Support for Games (NetGames). 1-6. DOI : https://doi.org/10.1109/ NetGames.2017.7991543

[7] De-Yu Chen and Magda El-Zarki. 2018. Improving the quality of 3D immersive interactive cloud based services over unreliable network. In Proceedings of the 10th International Workshop on Immersive Mixed and Virtual Environment Systems (MMVE'18). ACM, New York, 28-33. DOI : https://doi.org/10.1145/3210438.3210440

[8] Seong-Ping Chuah and Ngai-Man Cheung. 2014. Layered coding for mobile cloud gaming. In Proceedings of the International Workshop on Massively Multiuser Virtual Environments (MMVE'14). ACM, New York, Article 4, 6 pages. DOI : https://doi.org/10.1145/2577387.2577395 
[9] M. Claypool, D. Finkel, A. Grant, and M. Solano. 2012. Thin to win? Network performance analysis of the OnLive thin client game system. In Proceedings of the 11th Annual Workshop on Network and Systems Support for Games (NetGames). 1-6. DOI : https://doi.org/10.1109/NetGames.2012.6404013

[10] Cyril Crassin, David Luebke, Michael Mara, Morgan McGuire, Brent Oster, Peter Shirley, Peter-Pike Sloan, and Chris Wyman. 2015. CloudLight: A system for amortizing indirect lighting in real-time rendering. Fournal of Computer Graphics Techniques (FCGT) 4, 4 (15 Oct. 2015), 1-27. http://jcgt.org/published/0004/04/01/.

[11] Eduardo Cuervo, Alec Wolman, Landon P. Cox, Kiron Lebeck, Ali Razeen, Stefan Saroiu, and Madanlal Musuvathi. 2015. Kahawai: High-quality mobile gaming using GPU offload. In Proceedings of the 13th Annual International Conference on Mobile Systems, Applications, and Services (MobiSys'15). ACM, New York, 121-135. DOI : https:// doi.org/10.1145/2742647.2742657

[12] Matthias Dick, Oliver Wellnitz, and Lars Wolf. 2005. Analysis of factors affecting players' performance and perception in multiplayer games. In Proceedings of 4th ACM SIGCOMM Workshop on Network and System Support for Games (NetGames'05). ACM, New York, 1-7. DOI : https://doi.org/10.1145/1103599.1103624

[13] Yong Xue Eu, Jermyn Tanu, Justin Jieting Law, Muhammad Hanif B Ghazali, Shuan Siang Tay, Wei Tsang Ooi, and Anand Bhojan. 2016. SuperStreamer: Enabling progressive content streaming in a game engine. In Proceedings of the 24th ACM International Conference on Multimedia (MM'16). ACM, New York, 737-738. DOI : https://doi.org/10.1145/ 2964284.2973827

[14] Michael Garland and Paul S. Heckbert. 1997. Surface simplification using quadric error metrics. In Proceedings of the 24th Annual Conference on Computer Graphics and Interactive Techniques (SIGGRAPH'97). ACM Press/Addison-Wesley Publishing Co., New York, 209-216. DOI : https://doi.org/10.1145/258734.258849

[15] Mahdi Hemmati, Abbas Javadtalab, Ali Asghar Nazari Shirehjini, Shervin Shirmohammadi, and Tarik Arici. 2013. Game as video: Bit rate reduction through adaptive object encoding. In Proceedings of the 23rd ACM Workshop on Network and Operating Systems Support for Digital Audio and Video (NOSSDAV'13). ACM, New York, 7-12. DOI : https:// doi.org/10.1145/2460782.2460784

[16] H. Hong, C. Hsu, T. Tsai, C. Huang, K. Chen, and C. Hsu. 2015. Enabling adaptive cloud gaming in an open-source cloud gaming platform. IEEE Transactions on Circuits and Systems for Video Technology 25, 12 (Dec. 2015), 2078-2091. DOI : https://doi.org/10.1109/TCSVT.2015.2450173

[17] Hugues Hoppe. 1996. Progressive meshes. In Proceedings of the 23rd Annual Conference on Computer Graphics and Interactive Techniques (SIGGRAPH'96). ACM, New York, 99-108. DOI : https://doi.org/10.1145/237170.237216

[18] Hugues Hoppe. 1997. View-dependent refinement of progressive meshes. In Proceedings of the 24th Annual Conference on Computer Graphics and Interactive Techniques (SIGGRAPH'97). ACM Press/Addison-Wesley Publishing Co., New York, 189-198. DOI : https://doi.org/10.1145/258734.258843

[19] H. Hoppe. 1998. Smooth view-dependent level-of-detail control and its application to terrain rendering. In Proceedings of Visualization '98 (Cat. No.98CB36276). 35-42. DOI : https://doi.org/10.1109/VISUAL.1998.745282

[20] Chun-Ying Huang, Kuan-Ta Chen, De-Yu Chen, Hwai-Jung Hsu, and Cheng-Hsin Hsu. 2014. GamingAnywhere: The first open source cloud gaming system. ACM Trans. Multimedia Comput. Commun. Appl. 10, 1s, Article 10 (Jan. 2014), 25 pages. DOI : https://doi.org/10.1145/2537855

[21] Kyungmin Lee, David Chu, Eduardo Cuervo, Johannes Kopf, Yury Degtyarev, Sergey Grizan, Alec Wolman, and Jason Flinn. 2015. Outatime: Using speculation to enable low-latency continuous interaction for mobile cloud gaming. In Proceedings of the 13th Annual International Conference on Mobile Systems, Applications, and Services (MobiSys'15). ACM, New York, 151-165. DOI : https://doi.org/10.1145/2742647.2742656

[22] J. Levenberg. 2002. Fast view-dependent level-of-detail rendering using cached geometry. In Proceedings of IEEE Visualization, 2002 (VIS 2002). 259-265. DOI : https://doi.org/10.1109/VISUAL.2002.1183783

[23] Marc Levoy. 1995. Polygon-assisted JPEG and MPEG compression of synthetic images. In Proceedings of the 22nd Annual Conference on Computer Graphics and Interactive Techniques (SIGGRAPH'95). ACM, New York, 21-28. DOI : https://doi.org/10.1145/218380.218392

[24] Xiaofei Liao, Li Lin, Guang Tan, Hai Jin, Xiaobin Yang, Wei Zhang, Bo Li, Xiaofei Liao, Li Lin, Guang Tan, Hai Jin, Xiaobin Yang, Wei Zhang, and Bo Li. 2016. LiveRender: A cloud gaming system based on compressed graphics streaming. IEEE/ACM Trans. Netw. 24, 4 (Aug. 2016), 2128-2139. DOI : https://doi.org/10.1109/TNET.2015.2450254

[25] Y. Liu, S. Dey, and Y. Lu. 2015. Enhancing video encoding for cloud gaming using rendering information. IEEE Transactions on Circuits and Systems for Video Technology 25, 12 (Dec. 2015), 1960-1974. DOI : https://doi.org/10.1109/TCSVT. 2015.2450175

[26] Leonard McMillan, Jr. 1997. An Image-based Approach to Three-dimensional Computer Graphics. Ph.D. Dissertation. University of North Carolina at Chapel Hill, Department of Computer Science.

[27] F. Messaoudi, G. Simon, and A. Ksentini. 2015. Dissecting games engines: The case of Unity3D. In Proceedings of the International Workshop on Network and Systems Support for Games (NetGames). 1-6. DOI : https://doi.org/10.1109/ NetGames.2015.7382990 
[28] S. Möller, S. Schmidt, and S. Zadtootaghaj. 2018. New ITU-T standards for gaming QoE evaluation and management. In Proceedings of the 10th International Conference on Quality of Multimedia Experience (QoMEX). 1-6. DOI : https:// doi.org/10.1109/QoMEX.2018.8463404

[29] A. K. Moorthy and A. C. Bovik. 2011. Blind image quality assessment: From natural scene statistics to perceptual quality. IEEE Transactions on Image Processing 20, 12 (Dec. 2011), 3350-3364. DOI : https://doi.org/10.1109/TIP.2011. 2147325

[30] S. Mowlaei, S. Schmidt, S. Zadtootaghaj, and S. Möller. 2018. Know your game: A bottom-up approach for gaming research. In Proceedings of the 10th International Conference on Quality of Multimedia Experience (QoMEX). 1-3. DOI : https://doi.org/10.1109/QoMEX.2018.8463423

[31] X. Nan, X. Guo, Y. Lu, Y. He, L. Guan, S. Li, and B. Guo. 2014. A novel cloud gaming framework using joint video and graphics streaming. In Proceedings of the IEEE International Conference on Multimedia and Expo (ICME). 1-6. DOI : https://doi.org/10.1109/ICME.2014.6890204

[32] I. Nave, H. David, A. Shani, Y. Tzruya, A. Laikari, P. Eisert, and P. Fechteler. 2008. Games@large graphics streaming architecture. In Proceedings of the IEEE International Symposium on Consumer Electronics. 1-4. DOI : https://doi.org/ 10.1109/ISCE.2008.4559473

[33] Andreas Petlund, Kristian Evensen, Pål Halvorsen, and Carsten Griwodz. 2008. Improving application layer latency for reliable thin-stream game traffic. In Proceedings of the 7th ACM SIGCOMM Workshop on Network and System Support for Games (NetGames'08). ACM, New York, 91-96. DOI : https://doi.org/10.1145/1517494.1517513

[34] Peter Quax, Patrick Monsieurs, Wim Lamotte, Danny De Vleeschauwer, and Natalie Degrande. 2004. Objective and subjective evaluation of the influence of small amounts of delay and jitter on a recent first person shooter game. In Proceedings of 3rd ACM SIGCOMM Workshop on Network and System Support for Games (NetGames'04). ACM, New York, 152-156. DOI : https://doi.org/10.1145/1016540.1016557

[35] S. Schmidt, S. Möller, and S. Zadtootaghaj. 2018. A comparison of interactive and passive quality assessment for gaming research. In Proceedings of the 10th International Conference on Quality of Multimedia Experience (QoMEX). 1-6. DOI : https://doi.org/10.1109/QoMEX.2018.8463417

[36] Shu Shi, Cheng-Hsin Hsu, Klara Nahrstedt, and Roy Campbell. 2011. Using graphics rendering contexts to enhance the real-time video coding for mobile cloud gaming. In Proceedings of the 19th ACM International Conference on Multimedia (MM'11). ACM, New York, 103-112. DOI : https://doi.org/10.1145/2072298.2072313

[37] Shervin Shirmohammadi. 2013. Adaptive streaming in mobile cloud gaming. IEEE COMSOC Multimedia Communications Technical Committee E-Letter (2013), 20-23.

[38] S. Wang and S. Dey. 2010. Rendering adaptation to address communication and computation constraints in cloud mobile gaming. In Proceedings of the IEEE Global Telecommunications Conference (GLOBECOM 2010). 1-6. DOI : https:// doi.org/10.1109/GLOCOM.2010.5684144

[39] Z. Wang and Q. Li. 2011. Information content weighting for perceptual image quality assessment. IEEE Transactions on Image Processing 20, 5 (May 2011), 1185-1198. DOI : https://doi.org/10.1109/TIP.2010.2092435

[40] Zhou Wang, H. R. Sheikh, and A. C. Bovik. 2002. No-reference perceptual quality assessment of JPEG compressed images. In Proceedings of the International Conference on Image Processing, Vol. 1. I-I. DOI:https://doi.org/10.1109/ ICIP.2002.1038064

[41] Andrew D. Wilson. 2017. Fast lossless depth image compression. In Proceedings of the 2017 ACM International Conference on Interactive Surfaces and Spaces (ISS'17). ACM, New York, 100-105. DOI: https://doi.org/10.1145/3132272. 3134144

[42] S. Zadtootaghaj, N. Barman, S. Schmidt, M. G. Martini, and S. Möller. 2018. NR-GVQM: A no reference gaming video quality metric. In Proceedings of the IEEE International Symposium on Multimedia (ISM). 131-134. DOI : https://doi.org/ 10.1109/ISM.2018.00031

[43] S. Zadtootaghaj, S. Schmidt, and S. Möller. 2018. Modeling gaming QoE: Towards the impact of frame rate and bit rate on cloud gaming. In Proceedings of the 10th International Conference on Quality of Multimedia Experience (QoMEX). 1-6. DOI : https://doi.org/10.1109/QoMEX.2018.8463416

[44] Liang Zhang and W. J. Tam. 2005. Stereoscopic image generation based on depth images for 3D TV. IEEE Transactions on Broadcasting 51, 2 (June 2005), 191-199. DOI : https://doi.org/10.1109/TBC.2005.846190

Received December 2018; revised May 2019; accepted May 2019 